MATEC Web of Conferences 11,01020 (2014)

DOI: $10.1051 /$ matecconf / 20141101020

(C) Owned by the authors, published by EDP Sciences, 2014

\title{
La perméabilité relative aux gaz : Une propriété de transport importante contrôlant la durabilité du béton partiellement saturé.
}

\author{
Zine El-Abidine Kameche ${ }^{1}$, Fouad Ghomari ${ }^{1}$, Marta Choinska ${ }^{2}$, Abdelhafid Khelidj ${ }^{2}$ \\ ${ }^{1}$ Université de Tlemcen, Département de génie civil, Tlemcen, Algérie \\ ${ }^{2}$ Université de Nantes, Institut de Recherche en Génie Civil et Mécanique, Saint-Nazaire, France
}

\begin{abstract}
Résumé. Les propriétés de transport du béton, principalement la perméabilité, jouent un rôle clé dans l'évaluation et la prévision de la durabilité des structures en béton armé. En effet, la perméabilité aux gaz est un indicateur majeur pour évaluer la capacité du matériau à résister à la pénétration d'espèces chimiques agressives. Toutefois, l'aptitude du béton à se faire pénétrer par ces agents agressifs, ne peut pas toujours suivre une loi Darcienne puisque ce matériau n'est que très rarement saturé dans la réalité. En effet, la perméabilité du béton ordinaire, de nature poreuse, dépend du taux d'humidité qui fluctue dans ses interstices en fonction du temps en relation avec son milieu environnant. L'objectif de nos travaux est ainsi d'étudier la perméabilité du béton ordinaire partiellement saturé, confectionné à l'aide de deux types de ciment (CEM II/A 42,5 et CEM II/A 32,5), en soulignant l'attention particulière à l'influence de la taille du corps d'épreuve sur la mesure de la perméabilité (effet d'échelle). Dans ce but, le béton est caractérisé en premier lieu par sa perméabilité intrinsèque, évaluée par l'application de la méthode de Klinkenberg, et la variation de ce paramètre est étudiée en variant le taux de saturation dans des éprouvettes cylindriques de tailles différentes et en s'intéressant également à l'influence des deux ciments différents sur l'évolution de la perméabilité aux gaz du béton. L'analyse des résultats obtenus met en évidence l'évolution sensible de la perméabilité relative aux gaz du béton ordinaire en fonction du degré de saturation des différents corps d'épreuves testés. Ces résultats expérimentaux obtenus sont susceptibles d'être introduits dans des modèles de prévision des transferts dans le béton et de la prédiction de la durabilité des ouvrages en béton. Dans ce cadre, la validité de la loi de Van Genuchten-Mualem pour les milieux poreux non saturés a été vérifiée pour les échantillons testés. Les résultats analytiques obtenus avec ce modèle semblent être satisfaisantes pour un béton ordinaire. En revanche, cette formule analytique de Van Guenuchten modifiée ne semble pas appropriée pour les différentes tailles des éprouvettes testées en béton ordinaire pour différents taux de saturation.
\end{abstract}

\section{Introduction}

Dans les structures en béton, la durabilité du béton et la corrosion de l'acier d'armature sont intimement liés à la perméabilité des surfaces en béton exposées [1]. Par conséquent, la perméabilité, définie comme le mouvement d'un fluide dans un milieu poreux sous une charge de pression appliquée, est la propriété la plus importante du béton régissant sa durabilité à long terme [2]. Donc, la perméabilité aux gaz des bétons est considérée comme un indicateur majeur de la durabilité des ouvrages en béton armé, car elle régit la pénétration d'agents agressifs gazeux, comme le dioxyde de carbone ainsi que le transfert de vapeur d'eau liée au séchage du matériau [3]. Cependant, son aptitude à se faire pénétrer par des agents agressifs varie de façon sensible en fonction du taux d'humidité (degré de saturation) qui peut évoluer dans le temps.

Plusieurs travaux ont été réalisés afin de développer des procédures expérimentales pour la détermination de la perméabilité aux gaz des matériaux cimentaires liées à leur teneur en humidité [4]. Coussy et al [5], ont en effet montré que l'évolution des propriétés physicomécaniques des matériaux cimentaires dépend très fortement de leur état de saturation. L'état hydrique du béton est très important parce que, selon le taux de saturation du matériau, différentes tailles de pores sont disponibles [6]. Ainsi, la perméabilité au gaz d'une éprouvette de béton augmente quand le taux de saturation moyen diminue [7-8-9].

L'objectif de nos travaux est ainsi d'étudier la perméabilité relative au gaz du béton à différents degrés de saturation. Dans ce but, le béton est caractérisé en premier lieu par sa perméabilité intrinsèque (paragraphe II.2) et la variation de ce paramètre est étudiée contre la variation du degré de saturation du matériau. En soulignant l'attention particulière à l'effet de la taille du corps d'épreuve (effet d'échelle) sur la mesure de la perméabilité au gaz du béton en fonction du degré de saturation. Deux bétons ordinaire ont été testés qui sont avec à base de deux ciments différents (CPJ CEM II/A 
42,5 et CPJ CEM II/A 32,5), alors l'influence de types du ciment sur l'évolution de la perméabilité aux gaz en fonction de degré de saturation a été étudiée.

Pour les mesures des perméabilités intrinsèques Kint des éprouvettes de béton de diverses tailles, la méthode de Klinkenberg [10] a été appliquée à différents degré de saturation. Un préconditionnement a été appliqué par séchage afin de couvrir le domaine du taux de saturation du béton ordinaire étudié de l'état complètement saturé à l'état complètement $\sec \left(0 \leq S_{r} \leq 1\right)$. Il est donc possible de déduire de ces expériences une courbe $K_{r g}=K_{r g}\left(S_{r}\right)$, où $K_{r g}$ est la perméabilité relative au gaz qui est définit comme le rapport entre la perméabilité mesurée après un moment $\mathrm{t}$ de séchage $\left(S_{r}=x\right)$, à la perméabilité mesurée à l'état complètement $\sec \left(S_{r}=0\right)$ après plusieurs mois de séchage.

Les résultats expérimentaux obtenus sont susceptibles d'être introduits dans des modèles de prévision des transferts dans le béton et de la prédiction de la durabilité des ouvrages en béton [11]. Dans ce cadre, la validité de la loi de Van Genuchten modifiée pour les milieux poreux a été vérifiée.

\section{Programme expérimental}

\subsection{Matériaux et corps d'épreuve}

L'étude est réalisée sur deux bétons ordinaires, formulés avec les mêmes quantités des différents constituants granulaires mais à base de différents ciment (CPJ CEM II/A 42,5 et CPJ CEM II/A 32,5). Les deux ciments utilisés dans cette étude sont des ciments Portlands composés de la cimenterie de Béni Saf (Nord Ouest d'Algérie). Les différents granulats (sable et graviers) proviennent de la carrière de l'Entreprise Nationale des Granulats à Sidi Abdelli (Wilaya de Tlemcen). La composition et les caractéristiques physiques des deux bétons ordinaires étudiés sont présentées sur le tableau 1.

Tableau 1. Compositions et caractéristiques physiques des bétons ordinaires étudiés

\begin{tabular}{|c|c|c|}
\hline \multirow{2}{*}{ Constituants } & \multicolumn{2}{|c|}{ Quantités $\left(\mathbf{k g} / \mathbf{m}^{\mathbf{3}}\right.$ ) } \\
\cline { 2 - 3 } & $\begin{array}{c}\text { CPJ CEM II/A } \\
\mathbf{4 2 , 5}\end{array}$ & $\begin{array}{c}\text { CPJ CEM II/A } \\
\mathbf{3 2 , 5}\end{array}$ \\
\hline Ciment & 350 & 350 \\
\hline Gravier (16/25) & 533 & 533 \\
\hline Gravier (8/16) & 432 & 432 \\
\hline Gravier (4/8) & 144 & 144 \\
\hline Sable (0/4) & 690 & 690 \\
\hline Eau & 175 & 175 \\
\hline Rapport E/C & 0,5 & 0,5 \\
\hline Densité apparente & 2324 & 2324 \\
\hline Porosité à l'eau & $13,9 \%$ & $14,7 \%$ \\
\hline Rc (28j) & $35 \mathrm{MPa}$ & $32 \mathrm{MPa}$ \\
\hline
\end{tabular}

Des éprouvettes cylindriques de trois tailles différentes $(15 \times 30) \mathrm{cm},(11 \times 22) \mathrm{cm},(5 \times 10) \mathrm{cm}$ ont étaient utilisées dans cette compagne expérimentale. Les deux éprouvettes de $15 \mathrm{~cm}$ et $11 \mathrm{~cm}$ de diamètre ont été confectionnées dans des moules en acier et l'éprouvette de $5 \mathrm{~cm}$ de diamètre a été obtenue à l'aide de carottage. Toutes les éprouvettes du béton obtenues ont été sciées, à l'aide d'une tronçonneuse, pour obtenir des disques de la partie médiane, d'une épaisseur de $5 \mathrm{~cm}$ pour les essais de perméabilité.

\subsection{Dispositif de mesure de la perméabilité au gaz}

La perméabilité a été mesurée au laboratoire GeM à l'aide d'un perméamètre à charge constante de type CEMBUREAU [12]. L'ensemble de dispositif expérimental est présenté sur la Fig. 1. La procédure expérimentale est objet de la recommandation AFPCAFREM [13]. L'essai, consiste à soumettre un corps d'épreuve en béton, à une pression en amont $P_{i n j}$ constante jusqu'à la stabilisation de l'écoulement de gaz à travers le matériau (régime permanent), la pression en aval étant la pression atmosphérique $P_{a t m}$. Le débit du gaz est mesuré en amont et en aval de l'éprouvette par des débitmètres massiques qui convertissent le débit massique en un débit volumique équivalent. Le gaz utilisé est l'azote $\left(\mathrm{N}_{2}\right)$. L'application du principe de conservation de la masse à la loi de Darcy pour l'écoulement isotherme d'un gaz, considéré comme parfait, permet de déterminer la perméabilité apparente $K_{a}\left(\right.$ en $\left.\mathrm{m}^{2}\right)$ [14]. La perméabilité apparente $\left(K_{a}\right)$ est non seulement dépendante de la structure poreuse du matériau mais aussi de la pression moyenne du gaz percolant ;

$$
P_{\text {moy }}=\left(P_{\text {inj }} / P_{\text {atm }}\right) / 2 \text {. }
$$

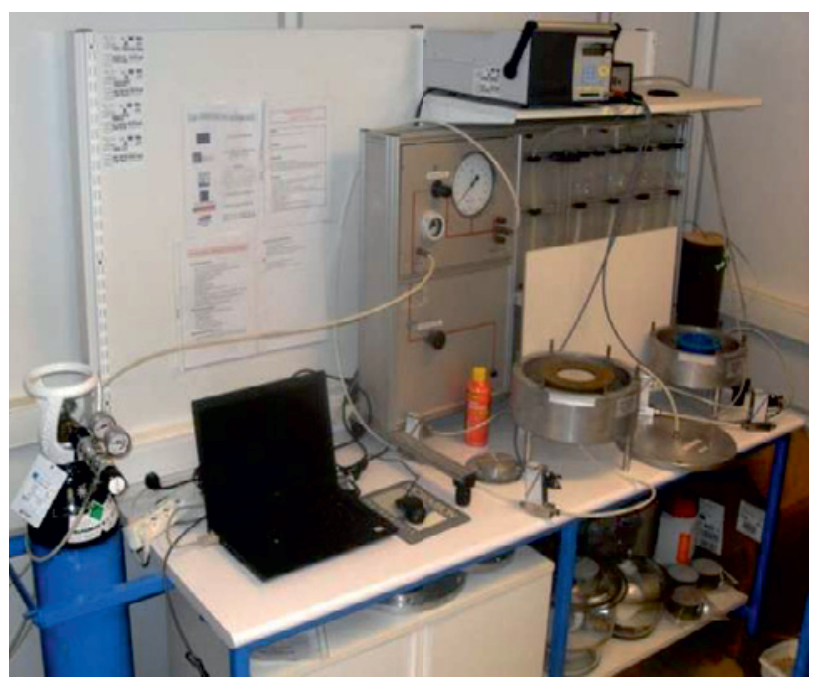

Fig.1. Dispositif expérimental de l'essai de la perméabilité au gaz (CEMBUREAU),

(Laboratoire GeM - IUT de St-Nazaire - Nantes)

Les mesures de la perméabilité ont été effectuées dans une salle à air conditionnées $(20 \pm 1){ }^{\circ} \mathrm{C}$. Chaque disque $\mathrm{du}$ béton a été testé à cinq pressions différentes : 0.1 , $0.15,0.2,0.25$ et $0.3 \mathrm{MPa}$. Une pression de confinement de $0.5 \mathrm{MPa}$ a été appliquée sur la surface latérale du 
corps d'épreuve en béton. Cette pression latérale doit être supérieure de la pression verticale appliquée $(0.3 \mathrm{MPa})$ pour assurer l'uni-direction du gaz percolant l'échantillon du béton.

Avant de prendre la mesure de débit du gaz, la pression du gaz percolant doit être appliquée environ 1 heure pour atteindre une stabilisation du flux à travers le corps d'épreuve et donc de mesurer la perméabilité au gaz du béton en régime permanent. Cette condition est vérifiée en prenant deux mesures séparées par un intervalle du temps de $10-\mathrm{min}$. Si deux valeurs diffèrent de moins de $2 \%$, le régime permanent est atteint et la mesure peut être enregistrée.

Dans le cas de écoulement laminaire d'un fluide compressible à travers les matériaux poreux, et pour chaque pression constante appliquée sur une face de l'échantillon du béton, la perméabilité apparente, appelée $\mathrm{Ka}$, peut être calculée directement par l'équation 1 , dérivée de la loi de Hagen-Poiseuille dans le cas de fluide compressible (gaz) [12] :

$K_{a}=\frac{2 \cdot \mu \cdot Q \cdot L \cdot P_{a t m}}{A\left(P_{i}^{2}-P_{a t m}^{2}\right)}$

Où: $Q$ est le flux du gaz $\left(\mathrm{m}^{3} / \mathrm{s}\right) ; A$ : Section de l'échantillon en contact avec le fluide percolant $\left(\mathrm{m}^{2}\right) ; L$ : L'épaisseur (dans le sens de flux du gaz) de l'échantillon (m); $\mu$ : coefficient de viscosité du gaz Nitrogen à $20{ }^{\circ} \mathrm{C}$ $\left(1.76 \times 10^{-5} \mathrm{~N} . \mathrm{s} / \mathrm{m}^{2}\right)$ for); $P_{i n j}:$ pression d'injection (pression appliquée absolue) $\left(\mathrm{N} / \mathrm{m}^{2}\right) ; P_{a t m}$ : Pression atmosphérique $\left(\mathrm{N} / \mathrm{m}^{2}\right)$.

Afin de déterminer la perméabilité intrinsèque du matériau, c'est-à-dire la perméabilité indépendante de la pression du gaz, il est nécessaire de réaliser plusieurs mesures à différentes pressions. Ainsi, la perméabilité apparente a été mesurée sous des gradients de pression compris entre 0,1 et $0,3 \mathrm{MPa}$ par palier de $0,05 \mathrm{MPa}$. Afin d'obtenir la valeur de la perméabilité intrinsèque $K_{\text {int }}$ qui ne correspond qu'à l'écoulement visqueux du fluide percolant, nous avons utilisé l'approche de Klinkenberg (1941) [10] qui détermine la perméabilité intrinsèque à partir d'une régression linéaire des différentes mesures de perméabilité apparentes effectuées pour différentes pressions d'injection selon l'inverse de la pression moyenne (moyenne entre la pression d'injection et la pression atmosphérique) [15].

La perméabilité intrinsèque $K_{\text {int }}$ est la valeur limite de la perméabilité apparente lorsque la pression moyenne du fluide tend vers l'infini, c'est-à-dire lorsque le gaz tend vers une phase condensée (liquide) [16]. Elle est définit comme suit :

$K_{a}=K_{\text {int }}\left(1+\frac{\beta}{P_{\text {moy }}}\right)$

$P_{m o y}=\frac{P_{0}+P_{a d m}}{2}$

Avec $\beta$ le coefficient de Klinkenberg, $P_{0}$ est la pression atmosphérique et $\beta$. $K_{\text {int }}$ est la pente de la droite de Klinkenberg. Cette méthode a été toujours la utilisée par plusieurs chercheurs (Bamforth [17]; Perraton \& al. [18]; Abbas \& al. [9]; Villain \& al. [11]).

\subsection{Préconditionnement}

Le préconditionnement est une procédure nécessaire afin de mesurer la perméabilité relative aux gaz du béton. Cette procédure doit être rapide et facile pour minimiser les modifications de la structure poreuse du matériau [13]. Dans ce but, plusieurs préconditionnements consistent à effectuer un séchage à $80^{\circ} \mathrm{C}$ à 28 jours et après à $105^{\circ} \mathrm{C}$ avant d'entamer l'essai de la perméabilité [13-19-11]. Cette procédure est rapide et très facile à effectuer mais elle modifie la structure poreuse du matériau [20-21] et peut provoquer des microfissures à cause d'un retrait due au séchage, ce qui augmente la perméabilité du béton. Pour cette raison, plusieurs études étaient pour objectif de développer des procédures expérimentales de préconditionnement pour déterminer la perméabilité aux gaz [3-9-15-19].

Après 28 jours de conservation des éprouvettes du béton, de différentes tailles, dans l'eau à une température de $20 \pm 2^{\circ} \mathrm{C}$, ces éprouvettes sont sciées pour obtenir des disques de la partie médiane, d'une épaisseur de $5 \mathrm{~cm}$. L'ensemble des corps d'épreuve des différents diamètres $(5,11$ et 15$) \mathrm{cm}$, sont séchés à $80^{\circ} \mathrm{C}$ par palier puis conservés afin d'obtenir différents taux de saturation. La durée de chaque palier équivaut $48 \mathrm{~h}$ de séchage dans une étuve ventilée, suivies de $24 \mathrm{~h}$ de refroidissement dans un dessiccateur afin d'atteindre une stabilisation thermique avant d'entamer l'essai de perméabilité. La surface latérale des corps d'épreuve est recouverte d'aluminium adhésif afin que les transferts gazeux, pendant le séchage et la mesure de la perméabilité, ne se produisent que par les deux faces planes des éprouvettes.

Les caractéristiques de ce préconditionne-ment ont été choisies, d'une part, pour limiter les risques de fissuration dus à de forts gradients de température, et d'autre part, pour obtenir des taux de saturation des éprouvettes les plus homogènes possibles. Etant donné l'épaisseur importante des éprouvettes $(5 \mathrm{~cm})$ et pour se rapprocher de l'état sec des éprouvettes testées, il nous a fallu plusieurs mois de séchage avant de prendre les pesées des éprouvettes, considérées comme les poids sec du matériau testé. La saturation complète des échantillons du béton a été effectuée sous vide en utilisant un dessiccateur et une pompe à vide suivant les recommandations AFREM. Des mesures de pertes de masse sont alors effectuées et les degrés de saturation des éprouvettes peuvent être ainsi déterminés.

\section{Analyse des résultats}

\subsection{Nature de l'écoulement du gaz pendant l'essai de perméabilité CEMBUREAU}

Les essais de perméabilité sont réalisés à pression constante, en régime permanent. Selon plusieurs auteurs (Perraton et al. [18]), (Villain et al. [11]), il est nécessaire de vérifier la nature de l'écoulement du gaz durant l'essai de perméabilité au gaz, car la détermination de la perméabilité intrinsèque selon Klinkenberg se place dans le cadre d'un écoulement visqueux. 


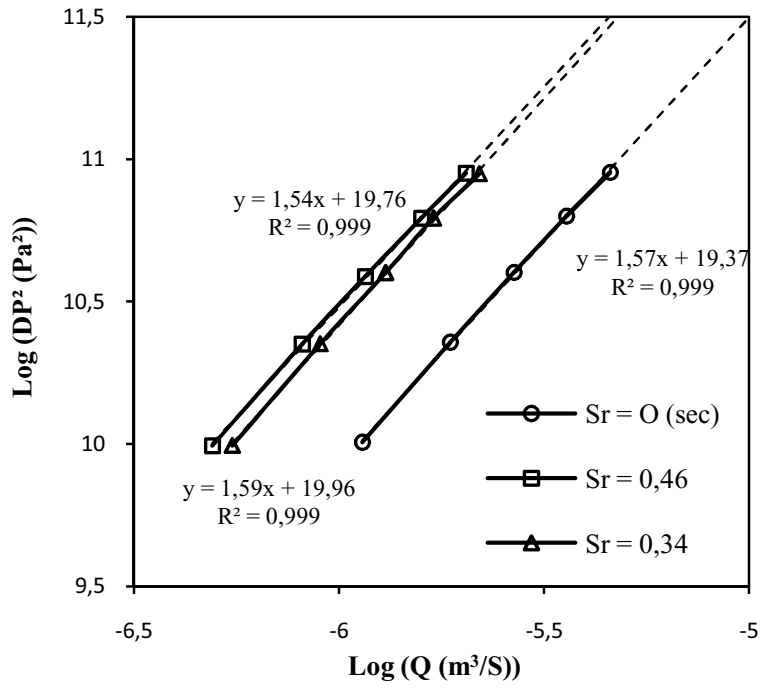

Fig. 2. Relation entre le carré du gradient de pression et le débit de gaz lors de l'essai de perméabilité

Selon (Perraton, 1992) [22], les point de mesure correspondant à un écoulement visqueux sont alignés ainsi que la pente de la droite doit être égale à 1 . Dans le cas d'une pression moyenne non constante durant l'essai et des forts débits gazeux, les points de mesure ne sont plus alignés et la courbe a une concavité tournée vers l'axe des abscisses (pente en tout point inférieure à 1) [11]. Nous avons appliqué cette méthode pour vérifier la nature de l'écoulement du gaz pour chaque éprouvette du béton testé dans cette étude. La figure. 2, présente une partie de ces résultats pour des corps d'épreuve de même taille et à différents degrés de saturation. Selon les courbes obtenues (Fig. 2), il est clair que la propriété de linéarité est bien respectée ainsi que la pente est toujours supérieure à 1 , ce qui est en accord avec les hypothèses de la méthode. On considère donc, que l'écoulement est visqueux et la perméabilité intrinsèque peut être approchée dans cette étude par la théorie de Klinkenberg.

\subsection{Résultats des essais de perméabilité au gaz}

Les premiers résultats des perméabilités intrinsèques présentées dans cette étude et qui correspondent à trois échantillons de béton à base du ciment portland composé (CPJ CEM II/A 42,5) sont présentés sur la figure. 3. Les corps d'épreuve testés sont de la même gâchée et de la même taille $(5 \mathrm{~cm}$ de hauteur et $15 \mathrm{~cm}$ de diamètre). Les perméabilités intrinsèque du béton sont exprimées en fonction de degré de saturation, mesurés par pesées après un préconditionnement appliqué par palier (décrit dans le paragraphe 2.3).

Les résultats montrent que la perméabilité est influencée par le degré de saturation en eau du béton. Ceci confirme les résultats obtenus par plusieurs chercheurs ; la perméabilité au gaz d'un corps d'épreuve en béton augmente quand le taux de saturation moyen diminue [9-15]. Les courbes des perméabilités intrinsèques en fonction de degré de saturation moyen peuvent être présentées par des courbes logarithmiques. La pente $\beta K_{\text {int }}$ de la droite de Klinkenberg en fonction de degré de saturation complète la caractérisation du béton testé [11]. Elle permet de calculer la perméabilité

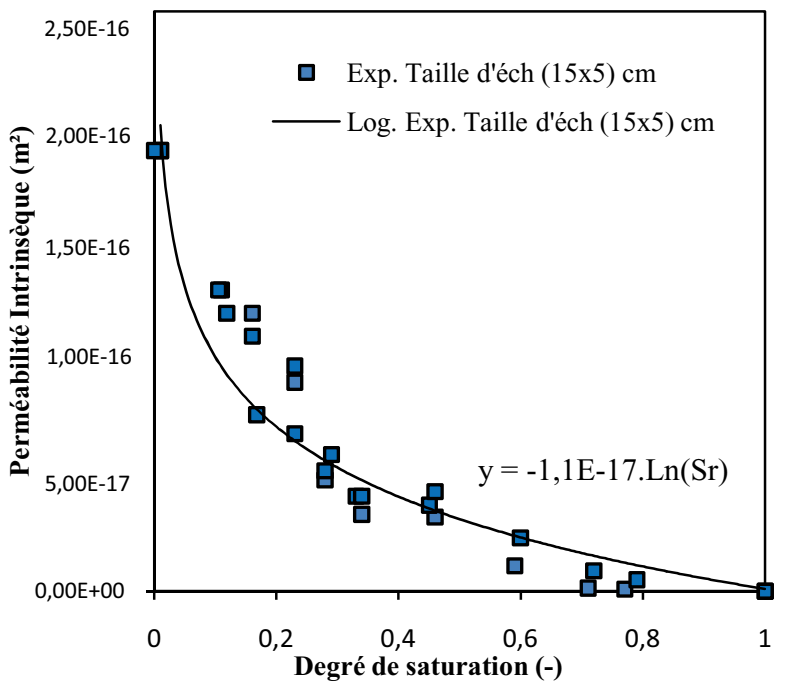

Fig. 3. Perméabilité intrinsèque du béton en fonction du degré de saturation

apparente du béton à une pression moyenne différente de celle utilisée pour la mesure à partir de l'équation (1).

À partir de ces mesures des perméabilités des éprouvettes du béton à différents taux de saturation, il est possible de déduire une courbe $K_{r g}=K_{r g}\left(S_{r}\right)$, où $K_{r g}$ est la perméabilité relative au gaz (Fig. 4). La perméabilité relative $K_{r g}$ peut être corrélée avec le degré de saturation. Alors, différentes relations ont été proposées par différents chercheurs [9-11] pour tenter de quantifier l'influence de la saturation en eau $S_{r}$ sur la perméabilité du béton.

Dans la littérature, on trouve une formule analytique (Equation (4)) qui correspond à la célèbre équation donnée par Van Guenuchten [23] pour l'évaluation de la perméabilité relative au gaz $K_{r g}$.

$K_{r g}\left(S_{r}\right)=\left(1-S_{r}\right)^{q} \cdot\left(1-S_{r}^{1 / m}\right)^{2 m}$

Cette relation est souvent admise pour la modélisation de la perméabilité d'un matériau poreux et en utilisant le modèle de Mualem [24] (avec $K_{r g}\left(S_{r}\right)=1$ quand $S_{r}=$ 0 , état sec) [25].

Dans cette formule, $m$ est un coefficient à déterminer à partir de la courbe de pression capillaire du matériau $P_{C}=P_{C}\left(S_{r}\right)$ [26]. A partir d'une série de mesures issues de la littérature, des valeurs de $q$ comprises entre 3.5 et 5.5 semblent être satisfaisantes [27]. La valeur de $q=5,5$ peut être généralisée pour les calculs de la perméabilité relative aux gaz de tous les matériaux cimentaires [28].

L'évolution de la perméabilité relative au gaz en fonction du degré de saturation en eau sera donc définie par l'équation (5):

$K_{r g}=\left(1-S_{r}\right)^{q} \cdot\left(1-S_{r}^{2}\right)$

La validité de cette loi de Van Genuchten-Mualem pour la modélisation de la perméabilité d'un matériau poreux a été vérifiée avec différentes valeurs de facteur $q$ (de $q=2,5$ jusqu'à $q=5,5$ ) avec les résultats obtenus expérimentalement avec notre béton ordinaire (Fig. 4). 


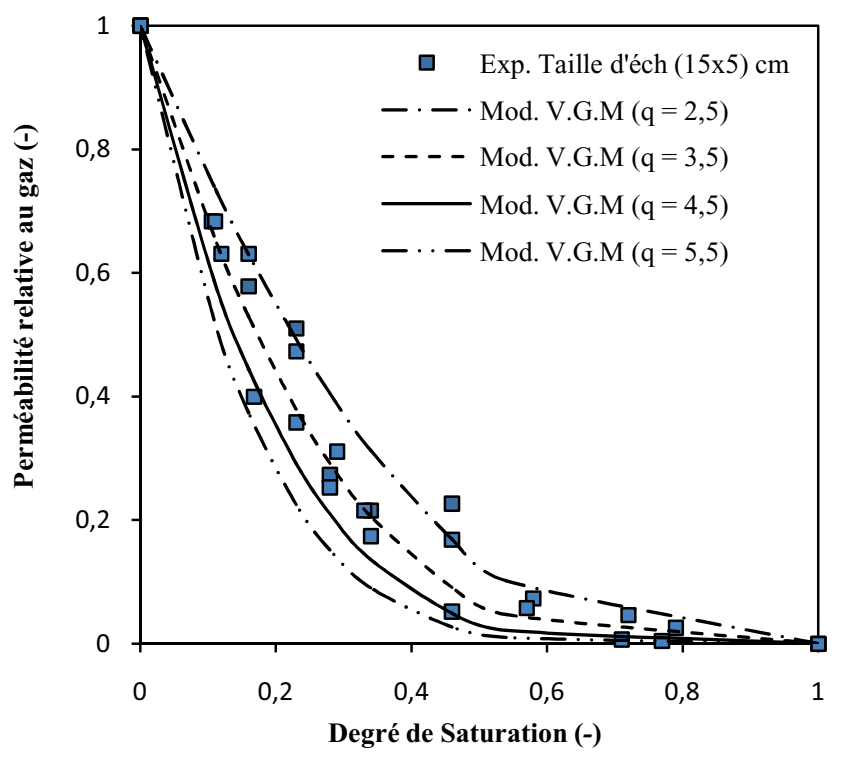

Fig. 4. Perméabilité relative au gaz du béton en fonction de degré de saturation.

Dans un intervalle restreint de variation de degré de saturation $\left(0<S_{r}<0,2\right)$, la perméabilité relative aux gaz décroit fortement pour atteindre une perméabilité au gaz d'environ $40 \%$ par rapport à sa perméabilité intrinsèque à l'état $\sec \left(S_{r}=0\right)$. Le modèle de Van Genuchten modifié s'ajuste presque parfaitement avec les résultats obtenus expérimentalement, avec les trois éprouvettes testées. Nous considérons que la valeur de $q=3,5$ est la plus appropriée notre béton ordinaire pour le calcul de $K_{r g}$ de en fonction de $S_{r}$ par l'équation 5. Au-delà de $60 \%$ de saturation, la perméabilité relative au gaz de notre matériau testé devient quasiment nulle. Ceci est en accord avec des études antérieures qui ont été menées, quantitatives ou qualitatives, pour mettre en évidence l'influence de cette variable (degré de saturation) sur les propriétés de transfert. Ainsi, Picandet et al. [29] montrent que quel que soit l'état mécanique et la formulation du béton (ordinaire $\mathrm{OC}$, haute performance HPC ou haute performance fibré HPFC), plus le matériau est sec et plus la perméabilité au gaz du béton augmente. La saturation en eau est donc une donnée influente pour la perméabilité.

\subsection{Influence de type du ciment sur la perméabilité au gaz du béton}

La figure 5 montre l'évolution de la perméabilité intrinsèque $\left(K_{\text {int }}\right)$ en fonction de degré de saturation de deux bétons ordinaires, de la même formulation, mais à base de deux différents types du ciment (CPJ CEM II/A 42,5 \& CPJ CEM II/A 32,5). Trois corps d'épreuve ont été testés pour chaque béton. Les valeurs présentées sur la figure 5, sont des valeurs moyennes des trois échantillons testés.

Pourtant que les perméabilités intrinsèques des deux bétons (à base de ciments différents), à l'état complètement sec, sont de même ordre de E-16, mais les résultats montrent que les corps d'épreuve en béton à base du ciment CPJ CEM II/A 32,5, présentent une

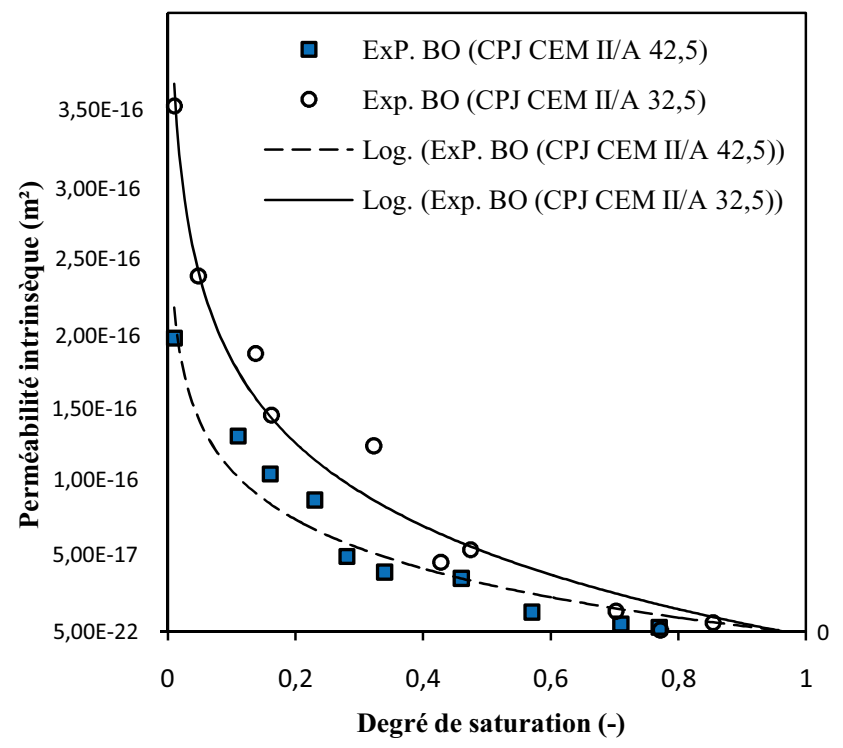

Fig. 5. Perméabilité intrinsèque du béton à base de différents ciments en fonction du degré de saturation

perméabilité au gaz, un peu plus importante que celle obtenue avec les échantillons du béton à base du ciment CPJ CEM II/A 42,5, pour des degrés de saturation, variant de l'état complètement $\sec \left(S_{r}=0\right)$ à l'état complètement saturé $(\mathrm{Sr}=1)$. Ceci peut être traduit par la porosité du béton à base du ciment CPJ CEM II/A 32,5 qui est relativement supérieure par rapport à la porosité du béton à base du ciment CPJ CEM II/A 42,5.

La perméabilité au gaz des deux bétons dépend fortement de degré de saturation. L'évolution de $K_{\text {int }}$ en fonction de $S_{r}$ des deux bétons ordinaires peut être tracée en fonction logarithmique, mais on constate qu'il est difficile d'écrire une seule et même loi pour tous les bétons.

La figure 6 montre l'évolution de la perméabilité relative au gaz $\left(K_{r g}\right)$ en fonction de degré de saturation $\left(S_{r}\right)$

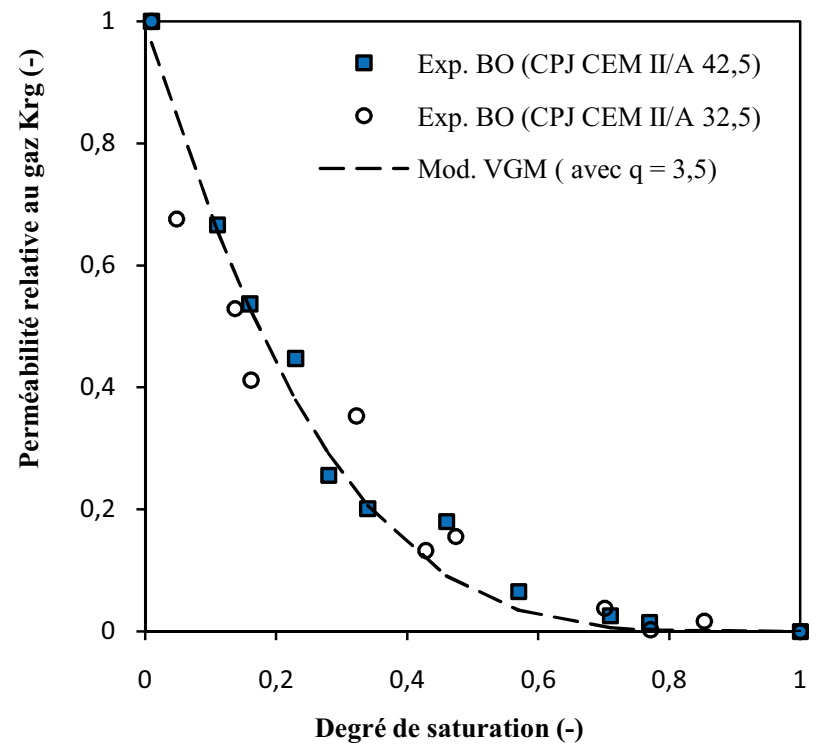

Fig. 6. Perméabilité relative au gaz des deux bétons ordinaire en fonction de degré de saturation. 
La relation de Van Genuchten-Mualem pour le calcul de la perméabilité relative aux gaz $K_{r g}$ en fonction de $S_{r}$, a été vérifiée. Les résultats expérimentaux, obtenus pour les deux bétons, s'ajustent presque parfaitement, surtout pour des valeurs de $S_{r}>0,5$ avec ce modèle dans le cas de $q=3,5$.

\subsection{Influence de la taille du corps d'épreuve}

Dans ce qui suit, nous avons étudié l'effet de la taille sur la mesure de la perméabilité relative de nos bétons ordinaires en fonction du taux de saturation.

\subsubsection{Résultats de la perméabilité des différents corps d'épreuves à base du Ciment CPJ CEM II/A 42,5}

La figure 7 présente l'évolution de la perméabilité intrinsèque $\left(K_{\text {int }}\right)$ des échantillons de différentes tailles et à base du ciment portland CPJ CEM II/A 42,5, en fonction de leurs degré de saturation. Dans cette partie d'étude, pour chaque degré de saturation, une moyenne arithmétique de trois mesures sur trois éprouvettes de même taille est présentée sur la figure 7 .

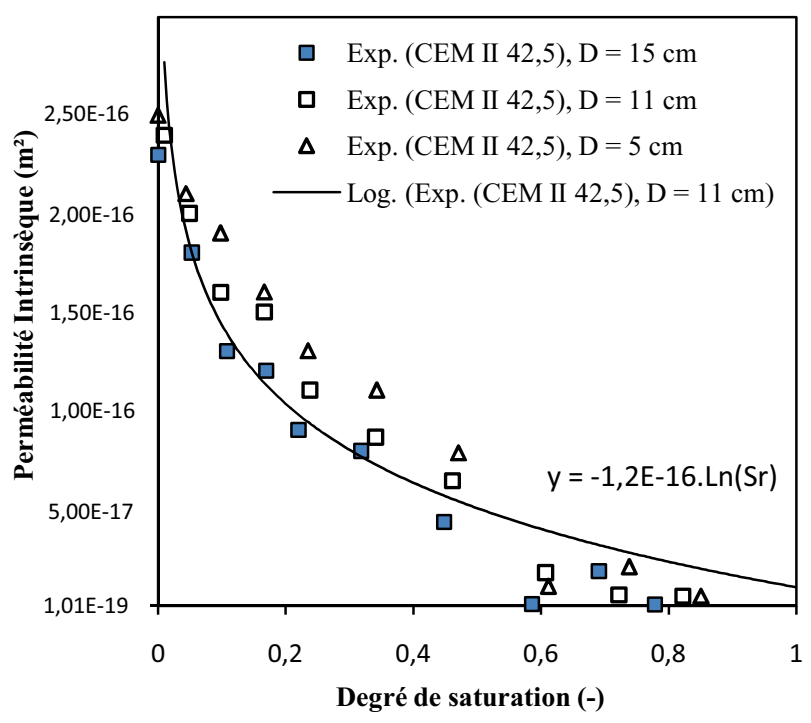

Fig. 7. Perméabilité intrinsèque des échantillons de différentes tailles en fonction de degré de saturation

Les résultats montrent que plus le corps d'épreuve est grand (de diamètre plus grand) plus la perméabilité mesurée est faible. Donc, la perméabilité intrinsèque du béton est, non seulement, influencée par son taux de saturation, mais aussi par la taille des corps d'épreuve. L'évolution de la perméabilité intrinsèque du béton entre l'état sec et $20 \%$ de saturation est importante.

Dans cet intervalle, la perméabilité de notre béton ordinaire passe de l'ordre de $\mathrm{E}^{-16} \mathrm{~m}^{2}$ à l'ordre de $\mathrm{E}^{-17} \mathrm{~m}^{2}$. Le préconditionnement a donc une grande influence sur la mesure, ainsi que la taille du corps d'épreuve du béton testé.

L'évolution de la perméabilité relative au gaz des corps d'épreuve de tailles différentes est présentée sur la figure 8. La validité de la loi de Van Genuchten-Mualem selon l'équation (5) a été vérifiée avec les perméabilités relatives obtenues avec les trois différents diamètres ainsi qu'avec différents coefficients $q$.

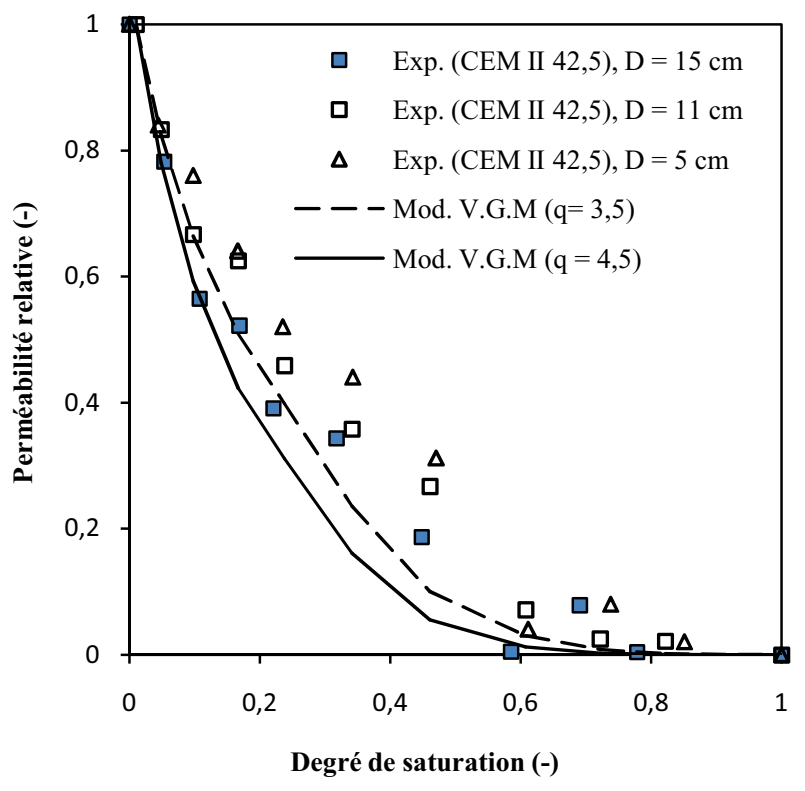

Fig. 8. Perméabilité relative au gaz des échantillons de différentes tailles en fonction de degré de saturation

Selon les courbes obtenues (Fig. 8), on observe une décroissance rapide de la perméabilité relative du matériau, entre l'état sec et $20 \%$ de taux de saturation. Alors, dans cette gamme de taux de saturation, on observe une grande influence de la perméabilité relative au gaz du béton ordinaire par le taux de saturation, ainsi plus le coefficient $q$ est inférieur (égal à 3,5), plus le modèle de Van Genuchten s'ajuste avec les résultats expérimentaux obtenus.

Au-delà du taux de saturation de $20 \%$ et à une gamme des $S_{r}$ intermédiaires, on observe un écart plus important et le modèle de Van Genuchten ne s'ajuste pas avec les résultats des perméabilités relatives obtenus expérimentalement.

La perméabilité relative au gaz du béton est influencée par la taille de l'échantillon testé. Nous pensons que le coefficient $q$ dépend, non seulement, de la nature du matériau mais aussi de la taille de l'échantillon.

\subsubsection{Résultats de la perméabilité des différents corps d'épreuves à base du Ciment CPJ CEM II/A 32,5}

La figure 9 présente l'évolution de la perméabilité au gaz des échantillons du béton de tailles différentes (différents diamètres ; $\mathrm{D}=15 \mathrm{~cm}$ et $\mathrm{D}=11 \mathrm{~cm}$ ) et à base du ciment CPJ CEM II/A 32,5.

Les résultats montrent que les valeurs de la perméabilité intrinsèque des corps d'épreuve de grande taille $(\mathrm{D}=15 \mathrm{~cm})$, obtenus pour des différents degrés de saturation, sont inférieures aux valeurs obtenus avec les corps d'épreuve de $11 \mathrm{~cm}$ de diamètre. On constate, d'après la figure que plus l'échantillon testé en béton, à base du ciment CPJ CEM II/A 32,5, est grand plus sa perméabilité diminue, ceci confirme les résultats obtenus ci-dessus avec le béton ordinaire à base du ciment CPJ CEM II/A 42,5. 


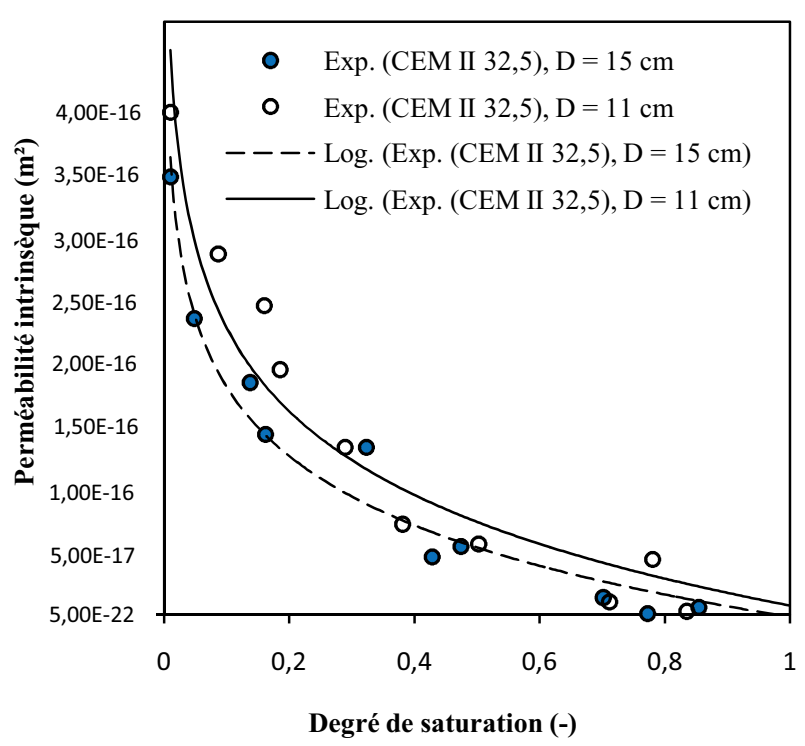

Fig. 9. Perméabilité intrinsèque des échantillons de différentes tailles en fonction de degré de saturation

L'évolution de $K_{r g}$ des corps d'épreuve de différentes tailles ( $15 \mathrm{~cm}$ et $11 \mathrm{~cm}$ de diamètre) et à base du ciment CPJ CEM II/A 32,5 en fonction $S_{r}$, est présentée sur la figure 10.

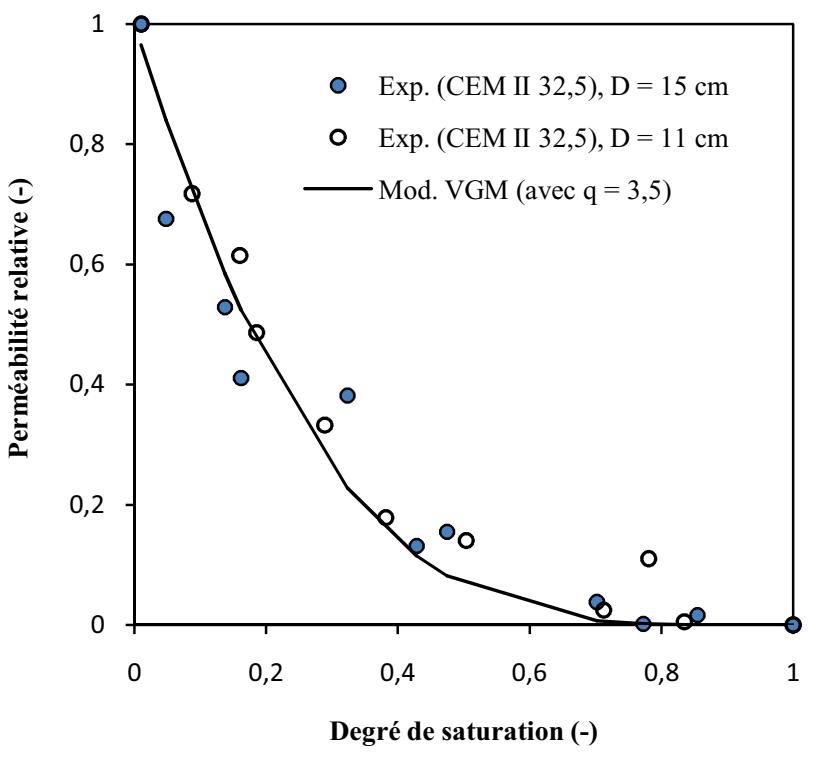

Fig. 10. Perméabilité relative au gaz des échantillons de différentes tailles en fonction de degré de saturation

Selon les résultats obtenus (Fig. 10), on considère que les perméabilités relatives aux gaz $\left(K_{r g}\right)$ sont influencés par la taille des échantillons du béton à base du ciment CPJ CEM II/A 32,5, pourtant qu'il y a un compromis acceptable pour la valeur de $q=3,5$ dans le calcul de $K_{r g}$ en fonction de $S_{r}$ par la relation de Van-GenuchtenMualem dans le cas de notre béton ordinaire,

\section{Conclusions}

Les perméabilités au gaz des différents corps d'épreuve en béton ordinaire, obtenues à différentes pressions d'admission, permettent de calculer la perméabilité intrinsèque selon l'approche de klinkenberg, qui est indépendante de la pression de mesure.

La perméabilité du béton ordinaire est fortement dépendante de son degré de saturation. Le préconditionnement appliqué (séchage à $80^{\circ} \mathrm{C}$ ) a donc une grande influence sur la mesure.

Le béton ordinaire à base du ciment portland composé CPJ CEM II/A 32,5 présente une perméabilité au gaz plus élevée de celle obtenue avec le béton ordinaire à base du ciment CPJ CEM II/A 42,5. Nous pensons que cela est due à une différence entre porosité des deux béton ordinaire étudiés.

L'originalité de cette étude a été de tester des corps d'épreuves en béton de diverses tailles pour avoir une estimation de l'effet d'échelle. Les résultats obtenus montrent que plus l'échantillon testé est grand de taille plus sa perméabilité diminue.

À partir des séries de mesures que nous avons effectuées, les résultats analytiques obtenus pour la perméabilité relative aux gaz avec des relations de Van Genuchten, qui sont basées sur les travaux de Mualem, à l'origine développée pour les sols non saturés, semblent être satisfaisantes pour un béton ordinaire, avec des valeurs de $q=3,5$ (souvent prise égale à 0,5 dans le cas des sols). En revanche, la formule analytique de Van Guenuchten modifiée ne semble pas appropriée pour les différentes tailles des éprouvettes testées en béton ordinaire et pour différents taux de saturation. Nous concluons que le coefficient $q$ dépend, non seulement, de la nature du matériau mais aussi de la taille de l'échantillon.

\section{References}

1. Aldea C.-M., Shah S. P., Karr A. Permeability of cracked concrete. RILEM Materials and Structures. vol. 32, PP. 370-376 (1999).

2. Banthia N., Biparva A., Mindess S. Permeability of concrete under stress. Cement and Concrete Reasearch, vol. 35, pp. 1651 - 1655. (2005).

3. Caré S., Derkx F. Determination of relevant parameters influencing gas permeability of mortars. Construction and Building Materials, vol. 25, pp. 1248 - 1256, (2011).

4. Boel V., Audenaert K., De Schutter G. Gas permeability and capillary porosity of self-compacting concrete. Mater \& Struct Vol 41, pp. 1283-1290, (2008).

5. Coussy O., Eymard R., Lassabatère T. Constituve Unsaturated modeling of drying deformable materials. Journal of Engineering Mechanics, 124 (6), pp. 658667, (1998).

6. Boroghel-Bouny V. Caractérisation des pâtes de ciment et des bétons. Méthodes, Analyses, Interprétations ». Edition Bulletin LCPC, Paris. (1994).

7. Parrott L. J., «Moisture Conditioning and transport properties of concrete test specimens $»$, Materials and structures, Vol. 27, pp 460-468, (1994).

8. Jacobs F., «Permeability to gas of partially saturated concrete », Magasine Concrete Research, Vol 50, n², pp 115-121, (1998). 
9. Abbas A., Carcasses M., Olivier J. P. Gas permeability of concrete in relation to it degree of saturation. Materials and Structures, vol. 32, pp. 3-8, (1999).

10. Klinkenberg J. The permeability of porous media to liquids and gases, in drilling and production practice, American Petroleum Institute, New York, pp. 200213, (1941).

11. Villain G., Baroghel-Bouny V., Kounkou C., Hua C. Mesure de la perméabilité aux gaz en fonction du taux de saturation des bétons. Revue Française de Génie Civil, Transfert dans les bétons et durabilité, vol. 5, pp. 251-268, (2001).

12. Kollek J. J. The determination of permeability of concrete to oxygen by the method CEMBUREAU - A recommendation. Materials and Structures. vol. 22, pp. 225-230, (1989).

13. AFREM, «Essai de perméabilité aux gaz des bétons durcis » : Mode opératoire recommandé par l'AFPCAFREM, Compte rendu des journées Techniques AFPC-AFREM «Durabilité des bétons 》, 11 et 12 Décembre, Toulouse, pp. 125 - 134, (1997).

14. Fabien A., Choinska M., Bonnet S., Pertué A., Khelidj A. Etude expérimentale sur l'effet de la taille des granulats sur le comportement mécanique et la perméabilité du béton. "TRANSFERT 2012". Mars (2012).

15. Picandet V. Influence d'un endommagement mécanique sur la perméabilité et sur la difusivité hydrique des bétons. Thèse de Doctorat, Université de Nantes, France, (2001).

16. Rozière E. Etude de la durabilité par une approcheperformantielle, Thèse de Doctorat de l'Ecole Centrale de Nantes, France, (2007).

17. Bamforth P.B. The relationship between permeability coefficients for concrete obtained using liquid and gas. Magazine concrete research, vol. 39, $\mathrm{n}^{\circ} 138$, pp. 3-11, (1987).

18. Perraton D., Aïtcin P-C., Carles-Gibergues A. Mesure de la perméabilité au gaz des bétons: perméabilité apparente et perméabilité intrinsèque Partie I : Validation des concepts de Carman et de Klinkenberg dans le cas d'un BHP. Bulletin des laboratoires des ponts et Chaussées, $\mathrm{n}^{\circ} 221$, pp. 6978, (1999).

19. Carcasses M., Abbas A., Olivier JP., Verdier J. An optimised preconditioning procedure for gas permeability measurement. Materials and Structures vol. 35, pp. 22-27, (2002).

20. Caré S. Effect of temperature on porosity and chloride diffusion in cement pastes. Construction and Building Materials, vol. 22, pp. 1560 - 1573, (2008).

21. Gallé C. Effect of dring on cement-based materials pore structure as identified by mercury intrusion porosimetry: a comparative study between ovenvacuum- and freeze-drying. Cem Concr Res vol. 31, pp. $1464-1477,(2001)$.

22. Perraton D. La perméabilité au gaz des bétons hydrauliques. Thèse de Doctorat de l'INSA de Toulouse, France, (1992).

23. Van Genuchten M.T. A closed form equation for Predicting the Unsaturated hydraulic conductivity of soils. Soil Science Society of America Journal, vol. 44, pp. 892-898, (1980).

24. Mualem Y. A New Model for Predicting the Unsaturated hydraulic conductivity of porous media. Water Resources Research, vol. 12, pp. 513-522, (1976).

25. Boroghel-Bouny V. Développement d'une approche globale, performantielle et predictive de la durabilité des structures en béton (armé) sur la base d'indicateur de durabilité; Billan et perspectives. LCPC Editions, Paris, Décembre (2008).

26. Coussy O., Baroghel-Bouny V., Dangla P., Mainguy M. Evaluation de la perméabilité à l'eau liquide des bétons à partir de leur perte en masse Durant le séchage, Revue Française de Génie civil, Numéro spécial «Transfert 2000», vol. 5, No. 1-2, (2001).

27. Jason L. Relation endommagement perméabilité pour les bétons: Application aux calculs des structures. Thèse de Doctorat de Ecole Centrale de Nantes, France, (2004).

28. Monlouis-Bonnaire JP., Verdier J., Perrin B. Prediction of the relative permeability to gas flow of cement- based materials. Cement and Concrete Research, vol. 34, pp. 737-744, (2004).

29. Picandet V., Khelidj A., Bastian G. Effect of axial compressive damage on gas permeability of ordinary and high performance concrete. Cement and Concrete Research, Vol. 31, pp. 1525-1532, (2001). 